\title{
Analysis of Medication Errors in Provincial Hospital
}

\author{
Hou Ning* and Tang Hui \\ Shandong Provincial Hospital, Shandong University, China
}

Received: August 24, 2017; Published: September 11, 2017

*Corresponding author: HOU Ning, Chief Pharmacist, Master's Supervisor of Shandong University, Research Fields: The Research of Pharmacy Administration Management \& Safe and rational use of drug, The Research of evaluation and analysis of drug utilization, China, Tel: 15168886165, Email: hou_ning@sina.com

Abbreviations: MEs: Medication errors; PAMRMI: Pharmacy Administration Management Regulations of Medical Institutions

\section{Introduction}

Drug is a key factor in patient safety events [1]. Medication errors (MEs) mean that may occur in the drug application, usually occur in the drug prescription, distribution, packaging, product labeling, dispensing, using and monitoring. Medication errors which are objective existence occur as a result of personal factors and/or system performance deficits [2,3]. The Pharmacy Administration Management Regulations of Medical Institutions (PAMRMI) definition of a medication error in China is as follows: "Any preventable event that may cause or lead to inappropriate medication use occurs in clinical medication." People usually focus on responsibility or punishment of physicians and pharmacists in the domestic medical related environment [4,5], while they don't attach great importance to the analysis of medication errors and don't develop the necessary measures to prevent the medication errors. People are used to hide problems, underreport and avoid responsibility, which makes medical safety somehow dangerous.

This study focuses on collecting medication errors of one first-class provincial hospital from 2012 to 2013 and analyzing the cause of error in order to make appropriate precautionary measures which could reduce errors and improve rational use of drug and ensure patients administration security. It is important to reduce the occurrence of medical disputes, maintain healthy and harmonious medical order, and improve the level of medical treatment and quality.

\section{Methods}

The study hospital was some first-class provincial hospital. The hospital pharmacy receives nearly 3.54 million medication orders (including outpatient prescription) and dispenses over 1350 million RMB medications during one-year period. We collected the medication errors prospectively between September 1, 2012 and August 31, 2013. The study was conducted mainly from 12 dispensaries of pharmacy department and some medical workers, although it didn't cover the whole medication errors. We used definitions from prior adverse drug reactions studies. MEs included errors during medication ordering, transcribing, dispensing, administering and/or monitoring. Suspected MEs and ADEs were collected by the way that the staffs reported positive and the chief of pharmacy supervised effectively. All the medication errors were categorized as harmful or not according to NCC MERP's Index [6,7] and other indexes such as type of error and cause of error. Serious MEs were analyzed for injury severity or potential severity.

\section{Results}

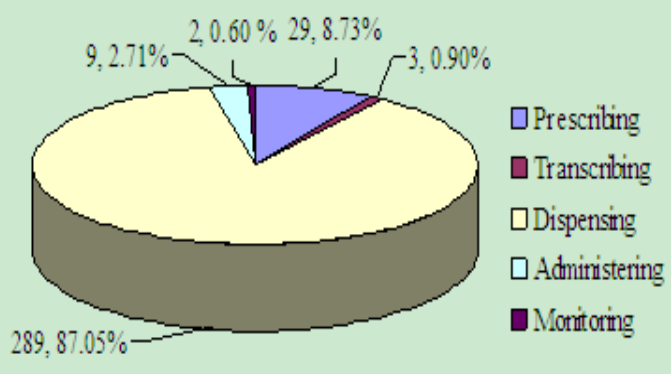

Figure 1: Node statistics.

A total of 332 cases were entered into the study. It's shown in (Table1), (Figure 1). There were 4 (1.20\%) cases of level A that were no error and 287 (86.45\%) of level B that the error did not reach the patient. We found 38 cases including 35 (10.54\%) of lever $C$ that reached the patient but did not cause patient harm and $3(0.91 \%)$ of lever D that were classified as errors that reached the patient and required monitoring to confirm that it resulted in no harm to the patient and/or required intervention to preclude harm. The severity of harm was significant $(0.91 \%)$ with medication errors that may have contributed to or resulted in temporary harm to the patient and required intervention. There were no fatal medication errors and the limit value of injuries was $0.90 \%$. 
Table 1: NCC MERP's Index for Categorizing Medication Errors.

\begin{tabular}{|c|c|c|c|}
\hline Category & Result of error & Percent \\
\hline & No Error & 4 & $1.20 \%$ \\
\hline A & Errcumstances or events that have the capacity to cause patient & 287 & $86.45 \%$ \\
\hline B & An error occurred ,but the error did not reach the patient & 35 & $10.54 \%$ \\
\hline C & An error occurred that reached the patient but did not cause patient harm & 3 & $0.90 \%$ \\
\hline D & $\begin{array}{c}\text { An error occurred that reached the patient and required monitoring to confirm that it resulted in } \\
\text { no harm to the patient and/or required intervention to preclude harm }\end{array}$ & 3 & $0.90 \%$ \\
\hline E & $\begin{array}{c}\text { An error occurred that may have contributed to or resulted in temporary harm to the patient and } \\
\text { required intervention }\end{array}$ & 3 \\
\hline F & $\begin{array}{c}\text { An error occurred that may have contributed to or resulted in temporary harm to the patient and } \\
\text { required initial or prolonged hospitalization }\end{array}$ & 0 & 0 \\
\hline G & An error occurred that may have contributed to or resulted in permanent patient harm & 0 & 0 \\
\hline H & An error occurred that required intervention necessary to sustain life & 0 \\
\hline I & An error occurred that may have contribute to or resulted in the patient's death & 0 \\
\hline
\end{tabular}

Table 2: Crosstab of error category index (multiple select).

\begin{tabular}{|c|c|c|c|c|c|c|c|}
\hline Type of error & A & B & C & D & $\mathbf{E}$ & Total & Percent \\
\hline Prescribing error & & 25 & 1 & & & 26 & $5.95 \%$ \\
\hline Wrong dispensing quantity & 2 & 129 & 15 & & & 146 & $33.41 \%$ \\
\hline Omission error & & & 10 & & & 44 & $10.07 \%$ \\
\hline Extra dose & 1 & 48 & 5 & & & 54 & $12.36 \%$ \\
\hline Wrong time & & & 0 & 1 & & 1 & $0.23 \%$ \\
\hline Wrong patient & & 5 & 3 & & & 8 & $1.83 \%$ \\
\hline Wrong administration route & & & & 1 & 2 & 3 & $0.69 \%$ \\
\hline Wrong dosage form or specifications & & 36 & 4 & 1 & & 43 & $9.84 \%$ \\
\hline Wrong drug & & 96 & 8 & 1 & & 105 & $24.03 \%$ \\
\hline Drug prepared incorrectly & 1 & 5 & & & & 6 & $1.37 \%$ \\
\hline Wrong administration technique & & & & & 1 & 1 & $0.23 \%$ \\
\hline Total & 4 & 380 & 46 & 4 & 3 & 437 & $100.00 \%$ \\
\hline
\end{tabular}

A total of 332 cases were categorized according to the type of medication errors (Table 2), (Figure 2). Approximately 75\% of medication errors were internal errors while internal error and out error ratio is 251: 81 . The wrong dispensing quantity which is higher was $33.41 \%$, and the wrong administration technique and wrong time have one case respectively. Four cases of error which involved the need to monitor the patients may be the wrong route, the wrong time, the wrong dosage form, the wrong drug specifications or the wrong drug. Three cases of error that resulted in temporary damage need treatment or intervention for patients may be the administration route or the drug administration technique in the medicine.

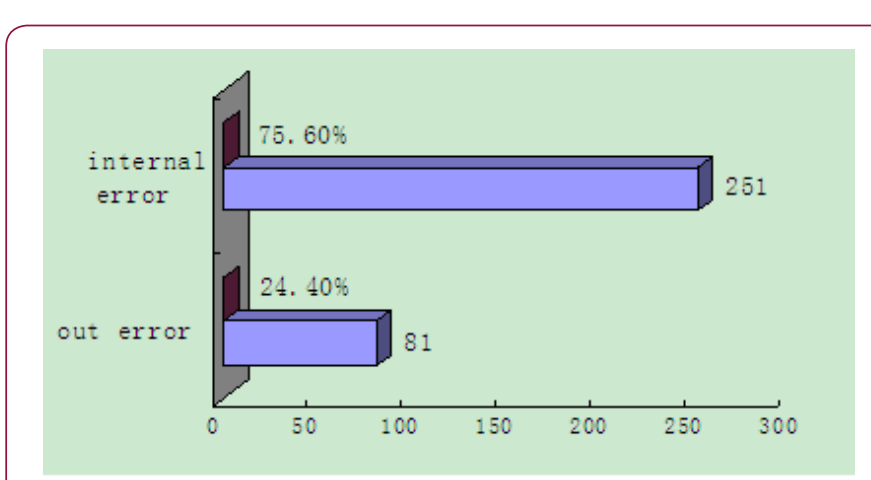

Figure 2: Internal errors and out error statistics. 
All the cases were categorized and analyzed according to the cause of error (Table 3). It's showed that prescribing error, performance deficit/half-heartedness and the same drug generic name were the main causes which accounted for $68.33 \%$. The Table 3: Cause of error (multiple select).

\begin{tabular}{|c|c|c|c|}
\hline Types & Cause of error & $\mathbf{n}$ & Percent \\
\hline \multirow[t]{4}{*}{ Human factor } & $\begin{array}{c}\text { Prescribing } \\
\text { (contraindication or off-label drug use) }\end{array}$ & 88 & $18.97 \%$ \\
\hline & Performance deficit/half-heartedness & 177 & $38.15 \%$ \\
\hline & Poor communication & 1 & $0.22 \%$ \\
\hline & Knowledge deficit & 23 & $4.96 \%$ \\
\hline \multirow[t]{5}{*}{ System factors } & Network fault/Information management system & 1 & $0.22 \%$ \\
\hline & Bugs & 1 & $0.22 \%$ \\
\hline & Computer entry/export & 2 & $0.43 \%$ \\
\hline & Drug dispensing system & 1 & $0.22 \%$ \\
\hline & Drug distribution process & 4 & $0.86 \%$ \\
\hline \multirow[t]{10}{*}{ Pharmaceuticals and environmental } & Drug storage proximity & 10 & $2.16 \%$ \\
\hline & Same generic names & 52 & $11.21 \%$ \\
\hline & Same brand names & 9 & \\
\hline & Dosage form confusion & 8 & \\
\hline & Different specification & 35 & \\
\hline & Similar packaging/labeling & 26 & $20.68 \%$ \\
\hline & Brand names look alike & 6 & \\
\hline & Similar generic names & 8 & \\
\hline & Names sounds alike & 12 & \\
\hline & Total & 464 & $100.00 \%$ \\
\hline
\end{tabular}

\section{Discussion}

We found that medication errors were common in the first-class provincial hospital, while there were fewer life-threatening and no fatal errors. Human factors, systems causes and environment conditions were judged to be most frequently primary cause (Table 3). We also found some near misses including 3 serious errors which resulted in temporary harm and required intervention to the patient. One was a patient developed severe pain and arm skin darker after intravenous injection of lyophilizing thrombin powder which should have been topical and forbidden to injection. Action is taken to prevent the adverse event immediately with subcutaneous injection of low molecular heparin sodium 5000U. We found that nurses with insufficient responsibility and not implementation of cheek systems maybe the main reason. Meanwhile, the original packaging of hemocoagulase atrox for injection and lyophilizing thrombin powder were look alike, and were deposited close to each other, which made pharmacist and nurse easily dispense wrong. The second medication error case was that a hospitalized patient was ordered and given by the riboflavin sodium phosphate which was added to sodium potassium magnesium calcium and glucose injection. After a moment, the patient found precipitation in the infusion. This is because the doctor shouldn't know the pharmaceutical incompatibility when they prescribe the drugs.

Meanwhile the system cannot provide technical support by which pharmacists can check prescription and nurses do not able similar packaging/labeling, brand names look alike, similar generic name drug and so on accounted for $20.68 \%$. The proportion of knowledge deficit and poor communication was 5.18\% and others only accounted for $4.11 \%$.

to be detect timely due to the lack of medical knowledge. The third case was that a patient with pregnant for three months treated with carboprost tromethamine injection, wrong intramuscular into intravenous drip, result in nausea, vomit and headaches, the adverse event was stopped by the physician timely and avoided serious consequences. Tracked the past medical records of this department we found that the doctors was used to overlook the medicine basis which should be shown in progress note. Second, doctors could not be trained effective or study drugs instruction themselves, which made them use drug inaccurately.

All the errors reminded that we must take action as soon as possible to keep continuous improvement in order to prevent or reduce medication errors. In terms of personnel, doctors and nursing staff must strictly implement the check system and turn it into jobs meanwhile they should improve their professional knowledge and skills so as to reduce human errors. We should increase training for rational use of drugs, strengthen drug oversight and ensure medication safety. All the doctors should be required to record all drugs in prescription and medical record. There should be a clear medicine basis and analysis in the progress note. In the field of management, pharmacy department and ward should store drugs separately and scientifically, meanwhile special medicines in pharmacy and ward should be marked obvious signs or alerted. Moreover, modern network supervision and warning method should be adopted, intercepting function of hospital information 
system should work as soon as possible, and pharmacist should play an important role in reviewing the doctor's advice. Drug manufacturers should be advised to design different drug packing which can be shown highlight instruction in accordance with different drug delivery.

It's known in (Figure $1 \& 2$ ) that dispensing accuracy of pharmacist played an impartment role in reducing medication errors. With the quickening pace of society, tedious and long-term work stress and tension, pharmacists easily made errors, while the lack of system execution, poor communication and some other external factors also could entail some potential dangers. Actually the professional regulate and standardization dispensing processes of pharmacists should remains to be improved.
It's known in (Tables $2 \& 3$ ) that wrong dispensing quantity $(146,33.4 \%)$ was the main error which was made by pharmacists in all medicine errors. By fishbone diagram of total quality management tools, wrong dispensing quantity was analyzed to find out the causes, which can help us take pertinence actions to solve the main reason in a short time and to prevent and control the occurrence of errors in a long time. It's shown clearly in (Figure 3) that pharmacists, tools and material were three most important related factors and each factors included some other reasons which can help us find the main object of solving. There also have other causes such as environment and imperfect process which made pharmacists errors.

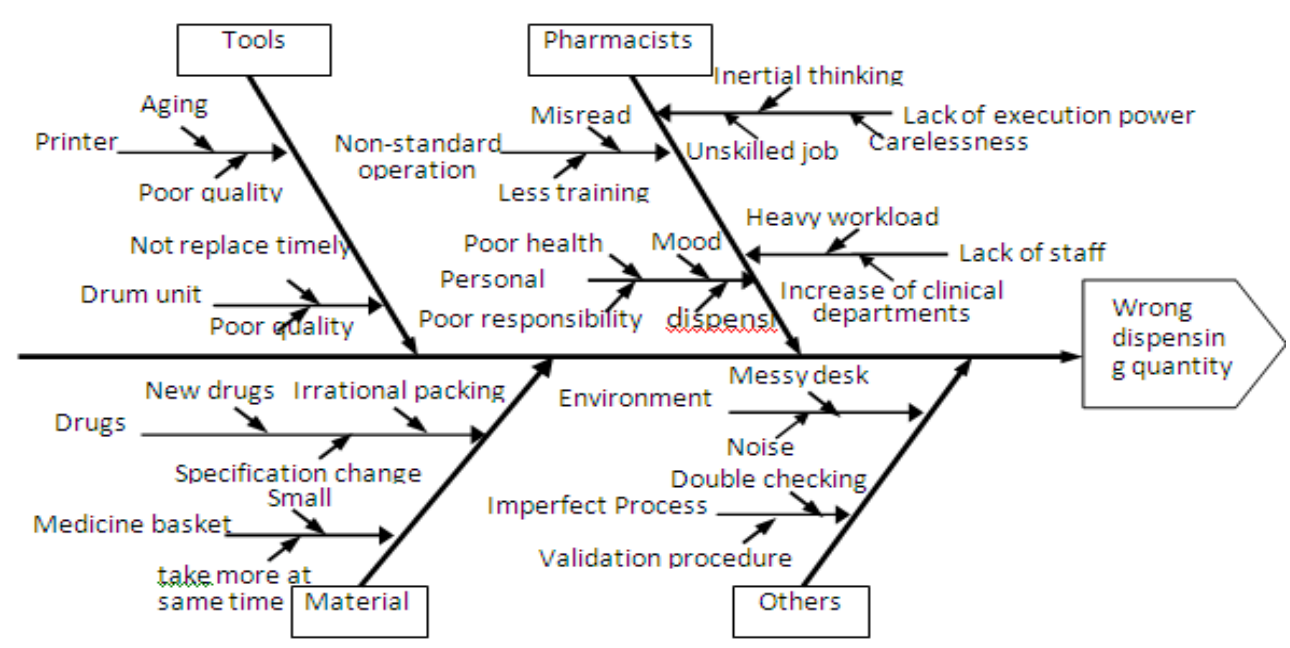

Figure 3: Fishbone Diagram of total quality management tools.

According to the analysis of fishbone diagram for cause of wrong dispensing quantity, effective measures should be taken continuously to prevent and reduce medicine errors. First, we should establish various regulations to meet hospital system running in standardized. Second, pharmacists should enhance responsibility and self-consciousness, improving their professional knowledge and skills, so as to master all the medicine operation instructions. If they found irrational prescription, they should communicate self-initiative with doctors. Third, all staff must strictly implement double check to intercepted the error as early as possible. All staff should be requested to report all medication errors and especially analyze the cause of errors with a greater incidence to preventing them from happening again. Fourth, pharmacy department should store drugs separately and scientifically, such as high-alert medications and other special medicines should be marked obvious signs and be standardized the ordering, storage, preparation, and administration. Finally, hospital should provide great working environment and arrange or increase appropriately staff in the rush hour, which may help us reduce medicine errors and promote better employee performance.

\section{Conclusion}

In conclusion, this study in some first-class provincial hospital showed that MEs are common and cause harm to patient but tend to be less life threatening and fatal. We overall analyzed the cause of wrong dispensing quantity error with the highest rates in pharmacists and take pertinence improvements using the total quality management tools. This study in the domestic still stays in a primary stage and medicine errors were collected by the only way that the staffs reported autonomously, so it inevitably has an influence on the objectiveness of the research to some extent. With the further development of China's medical reform and continuously absorbing and drawing lessons from foreign advanced management concept, hospital staff will aware of importance of report medicine errors. This research will be further combined with the actual situation to improve scientific and comprehensive the methods of obtaining the data. Additional studies are needed to determine which intervention strategies are most efficacious in this setting for preventing or reducing medication error and ensuring the safety and efficiency of drug use.

\section{References}

1. Kohn LT, Corrigan JM, Donaldson MS (2000) To Err Is Human: Building a Safer Health System, National Academy Press, Washington DC, Hong Kong.

2. Teinilä T, Kaunisvesi K, Airaksinen M (2011) Primary care physicians' perceptions of medication errors and error prevention in cooperation with community pharmacists. Research in Social and Administrative Pharmacy 7(2): 162-179. 
3. Aronson JK (2009) Medication errors: definitions and classification. Br Clin Pharmacol 67(6): 599-604.

4. Leape LL (1994) Error in medicine. JAMA 272(23): 1851-1857.

5. Reason J (2000) Human error: models and management. BMJ2000 320(7237): 768-770.
6. NCC MERP (2011) About Medication Error [EB/OL].

7. National Coordinating Council for Medication Error Reporting and Prevention. (1998) Taxonomy of medication errors. Rockville (MD): NCC MERP of the United States Pharmacopeia.

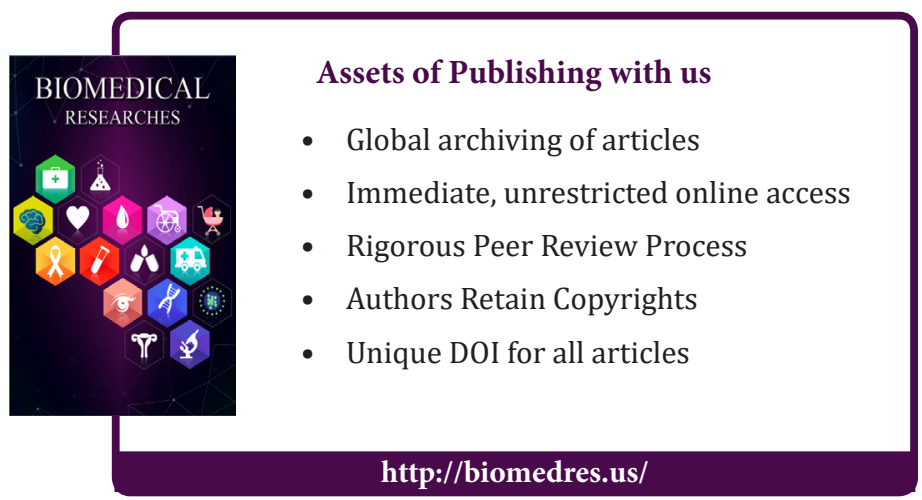

